\title{
Characterization of Collagen Peptides in Elaphuri Davidiani Cornu Aqueous Extract with Proliferative Activity on Osteoblasts Using Nano-Liquid Chromatography in Tandem with Orbitrap Mass Spectrometry
}

\author{
Yanjuan Zhai ${ }^{1}$, Zhenhua Zhu ${ }^{1}$, Yue Zhu ${ }^{1}$, Dawei Qian ${ }^{1, *}$, Rui Liu ${ }^{1, *}$, Yunru Peng ${ }^{2}$, \\ Yuhua Ding ${ }^{3}$, Zhen Ouyang ${ }^{4}$ and Jin-ao Duan ${ }^{1}$ \\ 1 Jiangsu Collaborative Innovation Center of Chinese Medicinal Resources Industrialization, \\ and National and Local Collaborative Engineering Center of Chinese Medicinal Resources \\ Industrialization and Formulae Innovative Medicine, Nanjing University of Chinese Medicine, \\ Nanjing 210023, China; zhaiyanjuan1990@126.com (Y.Z.); 04040416@163.com (Z.Z.); \\ nzyzy808@163.com (Y.Z.); dja@njucm.edu.cn (J.D.) \\ 2 Jiangsu Provincial Academy of Chinese Medicine, Nanjing 210028, China; pengyunru@126.com \\ 3 Jiangsu Dafeng Milu National Nature Reserve, Dafeng 224136, China; dingyuhuamilu@126.com \\ 4 School of Food and Biological Engineering, Jiangsu University, Zhenjiang 212013, China; \\ zhenouyang@ujs.edu.cn \\ * Correspondence: qiandw@njucm.edu.cn (D.Q.); cpulr@126.com (R.L.); \\ Tel./Fax: +86-25-8581-1916 (D.Q.); +86-25-8581-1524 (R.L.)
}

Academic Editor: Derek J. McPhee

Received: 3 October 2016; Accepted: 12 January 2017; Published: 20 January 2017

\begin{abstract}
First documented in Shennong Bencao Jing (about 200 B.C.-200 A.D.), Elaphuri Davidiani Cornu (EDC) has been recorded for its effects in strengthening bones and balancing other aspects of overall health for approximately 2000 years. In the present study, our aim was to investigate which are the components of the active EDC fraction by a peptidomic strategy. We explored the extent to which EDC increases the proliferation of osteoblasts by measuring the elevations in osteonectin and type I collagen mRNA levels and characterized it using nano-flow liquid chromatography in tandem with orbitrap mass spectrometry. In total, 272 peptide sequences from collagens were determined. "Hot regions" in parent proteins determined by peptide heat maps which indicated that amino acid sequences in the regions might undergo proteolysis easily and generate peptides. Among the identified peptides, $90.2 \%$ were hydrophilic, and the molecular weight of $97.1 \%$ of identified peptides was lower than $2000 \mathrm{Da}$. According to these results, EDC collagen-derived peptides were easily analyzed and identified. Moreover, this methodology is feasible to characterize the active peptides matrices originated from collagen hydrolysates or some other animal horn- derived TCMs.
\end{abstract}

Keywords: collagen peptide; Elaphuri Davidiani Cornu; nano-liquid chromatography-mass spectrum detection; osteoblast

\section{Introduction}

Animal horns are pointed projections on the head of animals, and are an important part of traditional Chinese medicines (TCMs). The antler of Milu deer (Elaphuri Davidiani Cornu, EDC) was first documented in Shennong Bencao Jing (about 200 B.C.-200 A.D.), and has reported efficacy in strengthening bones and balancing other aspects of overall health. However, most of the Milu deer in the Nanyuan Royal Hunting Garden of the Qing dynasty were killed, to be consumed by starving 
peasants in 1895 after the hunting garden was destroyed by a flood on the Yongding River. Then in 1900, the remaining Milu deer were shot and eaten by troops during the Boxer Rebellion, making Milu deer extinct in China [1]. Nowadays, as a species under first rank state protection in China, Milu deer are well protected. Since the reintroduction of Milu deer from the United Kingdom, the population has significantly increased from 18 to over 3000 in the last three decades in China [2]. The antlers of Milu deer are naturally shed every year, and can be easily collected, therefore, as easy obtained components, there were sufficient Milu deer antler resources for our scientific study.

Scientists are engaged in investigating the chemical components and pharmacological effects of EDC. Studies have shown that EDC contains amino acids, collagens, nucleosides, phospholipids, inorganic elements, etc. [2]. It has also been reported that EDC possesses diverse bio-activities, including anti-osteoporosis [3], anti-aging [4], and immuno-enhancing activity [5]. It is known that collagen plays an important structural function in organisms, especially in bone and cartilage. Collagen can be found in the bones, skin and connective tissue of animals. Collagen hydrolysates (CHs) are produced from collagen by breaking down the molecular bonds between peptides using chemical or enzymatic hydrolysis. CHs can improve bone metabolism and biomechanical parameters in ovariectomized mice [6]. Oral administration of $\mathrm{CHs}$ was demonstrated to increase the quantity of collagen and proteoglycans in the bone matrix of ovariectomized rats [7].

Like the antlers of other deer, such as the European red deer (Cervus Elaphus), EDC is a structure that regenerates every year. There are four different histological zones in a transversal cross section from the periphery to the center: the subvelvet zone, the zone of osteonic bone, the transition zone, and finally the central spongiosa zone [8]. The two outer regions are very dense and compact, whereas the center presents a general honeycombed structure [9]. As for other biomineralized tissues such as antler, bone, and, teeth, collagen is also the dominant protein in antler [10]. Traditional Chinese herbs are normally aqueous extracted and administered orally in clinical applications, which is typically the same for EDC. The EDC extract is a mixture of hundreds of compounds, most of which are peptides from non-specific proteolysis of collagen. It has been demonstrated that collagen peptides have an effect on bone resorption factors which supports the theory that they stimulate osteoblast activity [11]. However, a comprehensive peptide analysis of EDC extract is lacking therefore it is necessary to provide an overview of the peptide profile of the extract. Currently, proteins or peptides in a mixture can be identified using shotgun proteomics or peptidomics technology based on the generation of tandem mass spectrometry (MS/MS) [12]. In the present study, our aim is to investigate what composes the active EDC fraction by peptidomic strategy. Firstly, an active fraction of EDC (EDCF) was screened and the effects of EDCF on osteoblasts were evaluated. Subsequently, nano-flow liquid chromatography tandem orbitrap mass spectrometry (nano-LC-MS) was used to identify peptides in the fraction. We consider that this peptidomics-based approach is feasible to identify and analyze the active peptide matrix from animal-horn-derived TCMs.

\section{Results and Discussion}

\subsection{Inducing the Differentiation Activity of EDCF in Cultured Osteoblasts}

The osteoblasts were directly isolated from the calvarias of postnatal day 1 rats. The osteoblasts were able to undergo differentiation induced by dexamethasone and vitamin $\mathrm{C}$. The methylthiazole tetrazolium (MTT) method was used to observe the effect of ECDF on the proliferation of osteoblasts. As shown in Figure 1A, the proliferation of osteoblasts was up to $112 \%, 117 \%$, and $121 \%$ following incubation with EDCF at $0.01,0.03$, and $0.1 \mathrm{mg} / \mathrm{mL}$, respectively. The EDCF-induced osteoblast differentiation was further confirmed by activation of osteonectin and type I collagen (COL1A1) mRNA levels. Using quantitative real-time polymerase chain reaction (PCR), the levels of these differentiation markers increased significantly following treatment with dexamethasone and vitamin $C$ $(p<0.05 ; p<0.01)$. The levels of COL1A1 and osteonectin increased by more than 2.4-fold and 5.4-fold after treatment with EDCF $(p<0.05 ; p<0.01)$, respectively. 

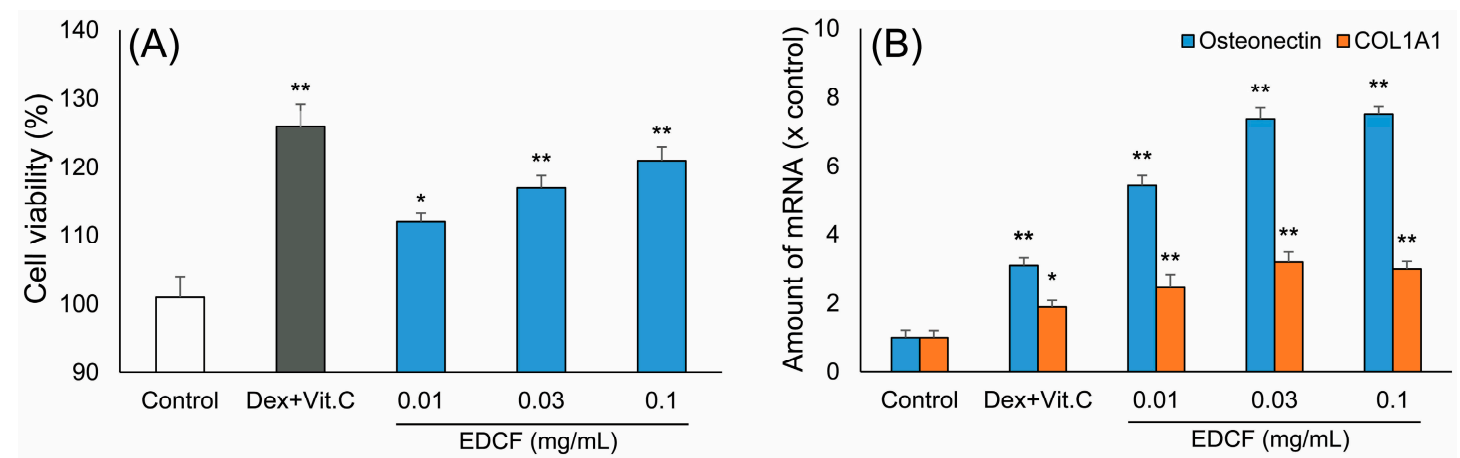

Figure 1. Proliferation of cultured osteoblasts induced by dexamethasone (Dex) and vitamin C (Vit. C), and by Elaphuri Davidiani Cornu (EDC) fraction. (A) Cell viability of osteoblasts; (B) Total mRNA levels of bone differentiation markers: type I collagen and osteonectin were quantified. Data were expressed as the fold of basal value ( $x$ basal) where the control value is set as $100 \%$ and 1 respectively, Mean \pm SEM, $n=3 .{ }^{*} p<0.05,{ }^{* *} p<0.01$.

The process of bone development can be divided into cell proliferation, cell differentiation, cell mineralization and cell apoptosis. This progression from one differentiation stage to the next is accompanied by the activation and subsequent inactivation of transcription factors and the expression of related marker genes i.e., osteopontin, collagen type I, alkaline phosphatase, bone sialoprotein and osteocalcin in osteoblasts [13]. Osteoblasts are differentiated to improve bone proliferation. The results of the present study indicated that EDCF had efficacy not only in promoting cell viability, but also in increasing mRNA expression of vital bone differentiation markers. These findings also suggest that EDCF could have potential effects on osteoporosis.

\subsection{Characterization of Peptides in EDCF}

Nano-LC MS/MS-based peptide identification has made peptide analysis in highly complex mixtures feasible. In the present study, taking the peptide LAGHHGDQGAPGAVGPAGPRGPAGP SGPAG as an example, high energy collision dissociation (HCD) was optimized to obtain $b$ and y ion series (Table S1, Figure 2A,B) and the error of these ions was lower than $0.02 \mathrm{Da}$ (Figure 2C). These ions were then searched against the Pecora database, and the amino acid sequence was confirmed. As shown in Figure 2A, it could be confirmed that this peptide matched with collagen $\alpha$-2(I) based on the database searching. As a result, a total of 272 peptides were identified in the EDCF, and the parent proteins of these peptides were collagens. It was confirmed that these peptides were hydrolyzed from these three parent proteins (unique peptides $\geq 2$ ), collagen $\alpha-1$ (W5P481) and collagen $\alpha-2$ (W5NTT7 and P02465).

Among these 272 peptides, the frequency of amino acid residues in each identified peptide was determined (Figure 3). The most frequent amino acid was glycine (Gly, G, 361 times), followed by proline (Pro, P, 215 times). Glycine and proline are the basic structural amino acid residues of collagens. A conspicuous Gly-X-Y repetitive sequence involves every third amino acid position occupied by a Gly and the $X$ and $Y$ positions are often occupied by Pro [14]. The total frequency of hydrophilic amino acids was 662, and the hydrophobic amino acids were observed 429 times (Figure 3A). Furthermore, the grand average of hydropathicity (GRAVY) index value was used to evaluate the hydrophilic and hydrophobic character of the identified peptides. As shown in Figure 3B, of the identified peptides, $90.2 \%$ were hydrophilic due to a GRAVY index value lower than 0 . In general, hydrophilic amino acids frequently occurred in the identified peptides of EDCF, which may appear hydrophilic in nature.

Considering the global peptides, as shown in Figure 3C, the molecular weight (MW) of identified peptides ranged from 793.40 to $3288.66 \mathrm{Da}$, and $97.1 \%$ of the peptides were lower than $2000 \mathrm{Da}$. Active peptides usually contain 2-20 amino acid residues per molecule therefore, there may be a higher chance that the identified peptides cross the membrane barrier and exert biological effects due to their low molecular weight [15]. 
sp|P02465|CO1A2_BOVIN Collagen alpha-2(I) chain

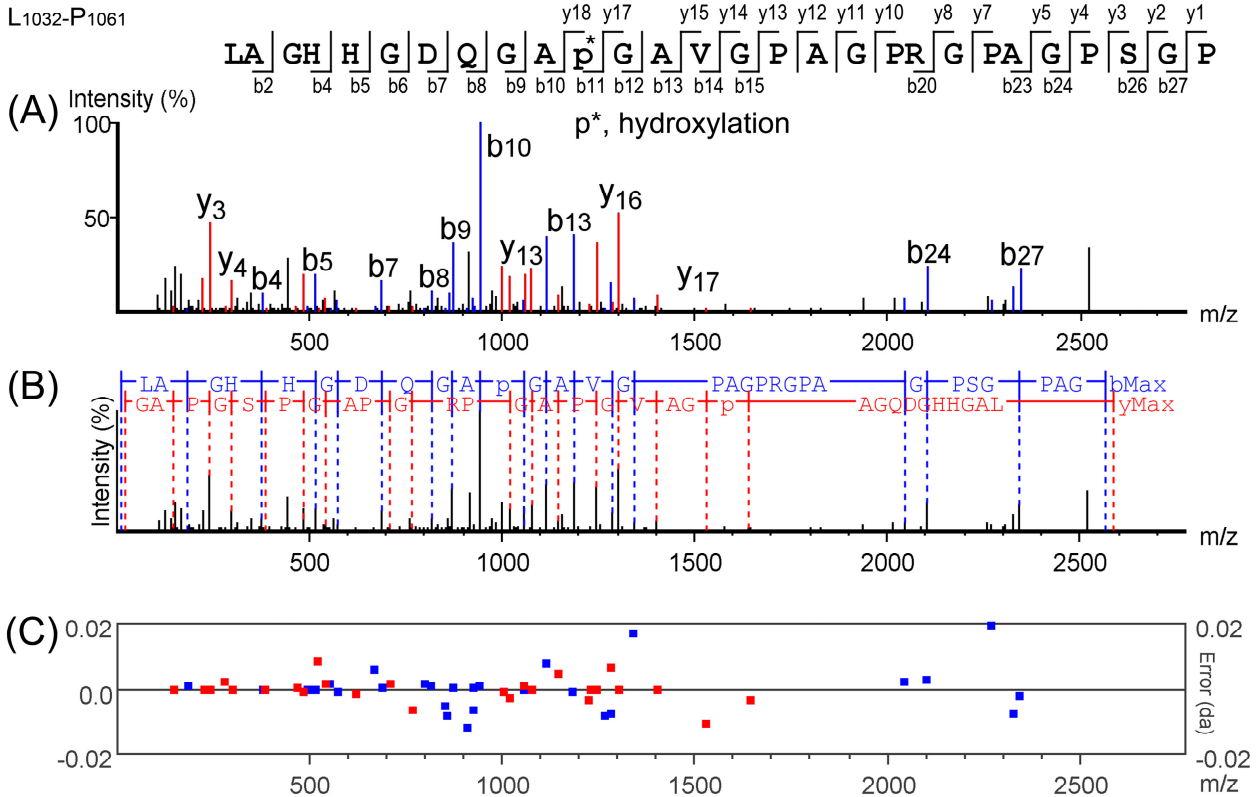

Figure 2. Identification of peptides based on the MS/MS spectra. (A) Amino acid sequence of the peptide and its MS/MS ions series, $\mathrm{b}$ ions and $\mathrm{y}$ ions were marked on the sequence of peptides; (B) Amino acid sequence calculated by de novo sequencing algorithm using PEAKS software; (C) Error of the ions. The mid line represents theoretical values of the ions, red spots represent the position of $\mathrm{y}$ ions, and blue spots represent the position of $\mathrm{b}$ ions. All errors are lower than $0.02 \mathrm{Da}$.
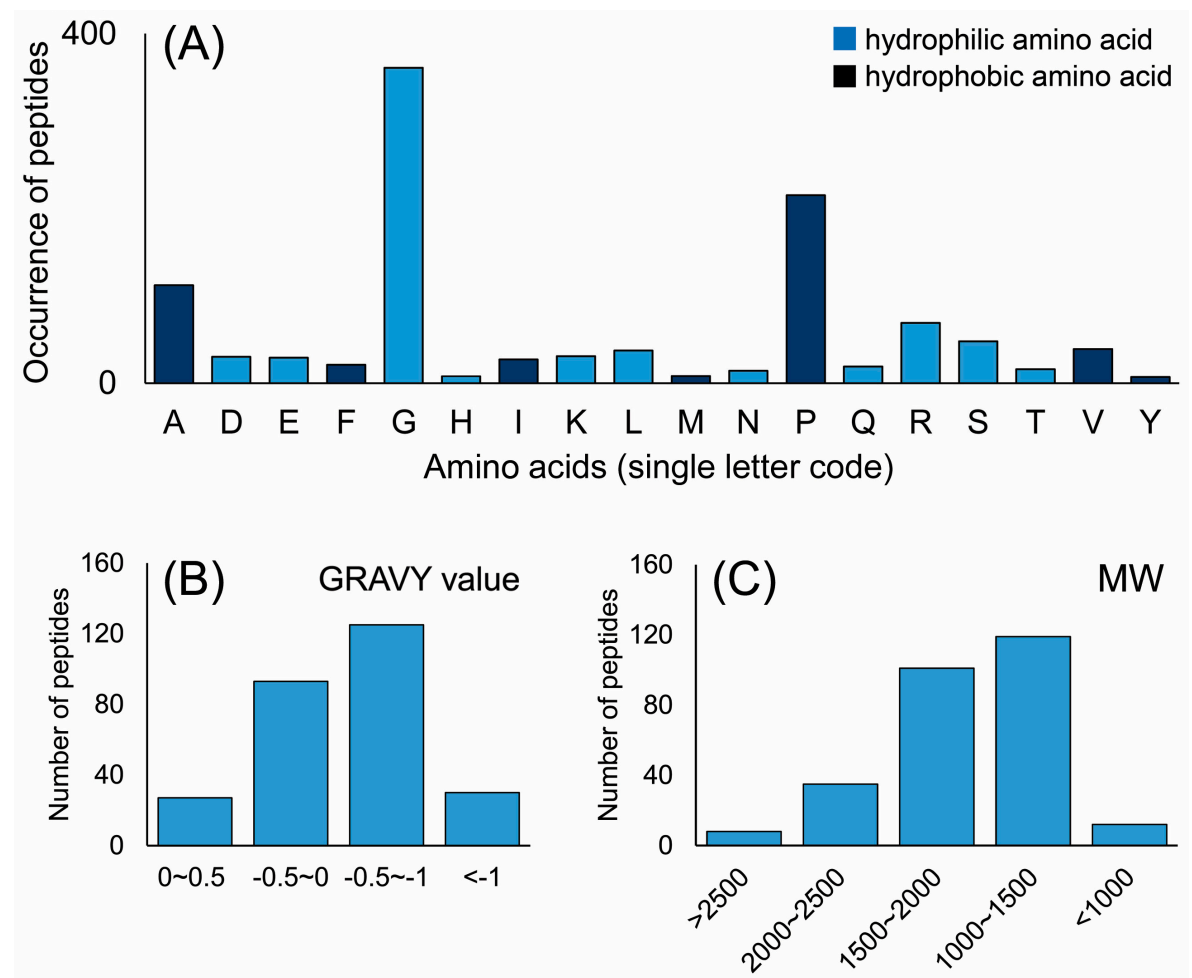

Figure 3. (A) Occurrence of the amino acids in all identified peptides; (B) Grand average of hydropathicity (GRAVY) index value of the identified peptides. GRAVY index values lower than 0 represent that the peptides possess hydrophilic properties; (C) Molecular weight (MW) distribution of the identified peptides. All of the value based on the total number of peptides. 
In the present study, a comprehensive analysis of the peptides was used to obtain information on the presence of peptides and the mode of peptide formation in EDCF. Primary sequences of peptides showed that most of these peptides originated from collagens, as shown in the Supplemental Data Figure S1, Figure S2 and Figure 4. MS/MS spectra of peptides were set up to search the Pecora database which contains 94,259 proteins (the Pecora are an infraorder of even-toed hoofed mammals with ruminant digestion, including deer). As a result, there are two parent proteins from which these peptides origin, collagen $\alpha-1$ (W5P481) and collagen $\alpha-2$ (W5NTT7 and P02465). It may be helpful to explain what happened to the EDC proteins during the aqueous extraction process. The regions which release peptides are not evenly distributed in the total protein sequences. In order to understand the locations of identified peptides, the number of times single amino acids made up the peptides in the primary sequence of proteins was counted and reported as a protein heat map. Heat maps of peptide distribution indicated the locations of highest hydrolysis of each protein, which graphically displayed the occurrence of the different amino acids in the primary sequence of the parent protein. In heat maps, as shown in Figure 4, there are particular "hot regions" in the amino acid sequence which might undergo proteolysis easily and generate peptides [16]. Blue regions indicate low occurrence, red regions indicate frequently appearing residues. In the primary sequence of proteins, each position only has one amino acid, and there were various hydrolyzed peptides which might be derived from the same protein fragment. Therefore, in the heat map, amino acids in each position would appear not only once. In contrast, there are also some amino acid sequences which do not undergo hydrolysis easily. As shown in Figure 4, according to the heat map profiles, three proteins showed different releasing types. Two types of collagen $\alpha-2(\mathrm{I})$ showed a similar profile (Figure 4 ).

P02465|CO1A2_BOVIN Collagen alpha-2(I)

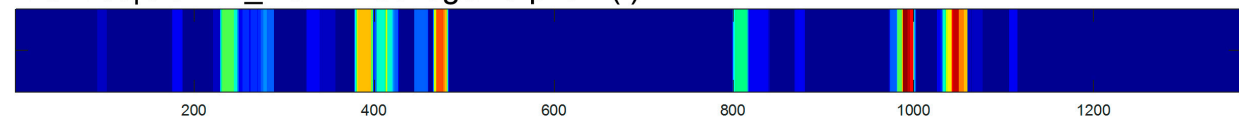

200

600

1200

W5NTT7_SHEEP Uncharacterized protein OS=Ovis aries $\mathrm{GN}=\mathrm{COL} 1 \mathrm{~A} 2$

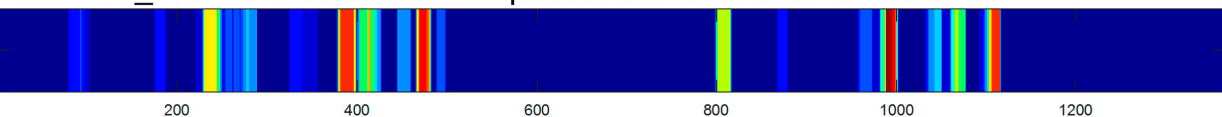

200

600

800

1200

W5P481_SHEEP Uncharacterized protein OS=Ovis aries GN=COL1A1

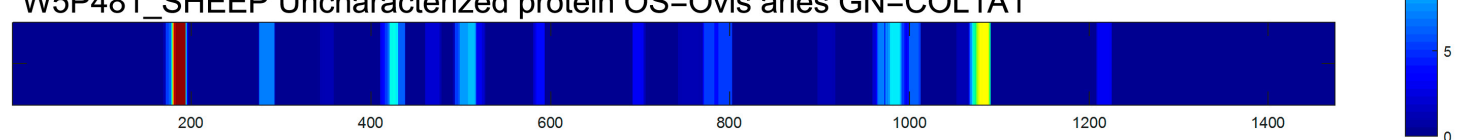

Figure 4. Heat maps of collagens. Heat maps graphically displaying the occurrence of the different amino acids in the primary sequence of the parent protein of the identified peptides. Blue regions indicate low occurrence; red regions indicate frequently appearing residues.

Taking collagen $\alpha$-2(I) as an example to explain how these peptides are generated, the locations of 120 peptides identified from collagen $\alpha$-2(I) are shown in Figure 5. Peptides were generated from seven main "hot regions" including R222 to G254, G255 to S289, R379 to G429, V466 to R484, G800 to V841, G974 to G1004, and G1028 to R1065, which were important sources of peptides. Water molecules normally occupy the space between collagens and interconnect them through hydrogen bonding [14]. As shown in Figure 5, the N-terminus and C-terminus amino acid residues of the seven "hot regions" were hydrophilic amino acids, except for V466 and V841. During the aqueous extraction process, non-specific cleavage usually occurred at hydrophilic amino acids. For example, it can be seen that the peptide fragment from R222 to G254 was first dropped from collagen and dissolved by water extraction, and a "hot region" was generated. The "hot region" was then irregularly hydrolyzed to generate peptides, such as RVGAPGPAGARGSDGSVGPVGPAGPIG, GARGSDGSVGPVGPAGPIG, and GARGSDGSVGPVGPA. 


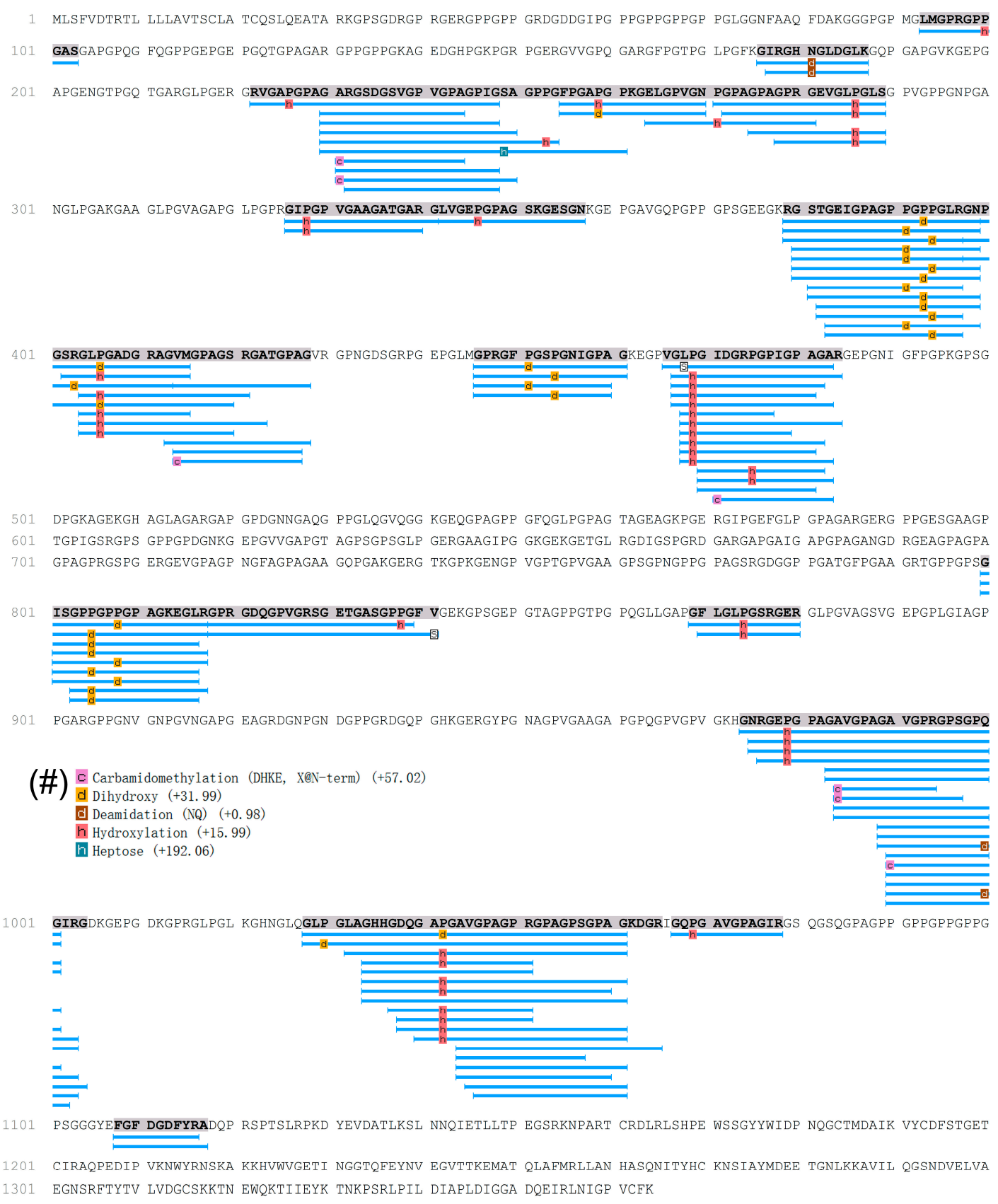

Figure 5. Sequence of collagen $\alpha$-2(I) (P02465 ICO1A2_BOVIN Collagen $\alpha-2(\mathrm{I})$ chain) and the distribution of identified peptides. (\#) Post-translational modification of proteins in collagen $\alpha$-2(I), "c": carbamidomethylation; orange " $d$ ": dihydroxy; brown " $d$ ": deamidation; pink " $h$ ": hydroxylation; and green " $h$ ": heptose.

Collagen is the main structural component in bone formation. Hu et al. has pointed out that fish collagen peptide has a positive effect on osteoblasts [13]. CHs have been widely used in clinical application, and a number of studies have suggested that $\mathrm{CHs}$ can improve bone collagen metabolism, bone mineral density and bone mass content in rats fed a calcium-deficient diet [17]. It was demonstrated that oral administration of $\mathrm{CHs}$ could reduce bone loss in the bone matrix by increasing the quantity of type I collagen and increasing bone strength in ovariectomized animals $[6,11,18]$. CHs with molecular weight lower than $3 \mathrm{kDa}$ can increase osteoblast proliferation, alkaline phosphatase activity, and COL1A1 gene expression. Similarly, the active fraction $(<3 \mathrm{kDa})$ of EDC promoted osteoblast proliferation and increased the gene expression of two important markers, osteonectin and COL1A1. 
The fragments of enzymatic CHs may be taken up by the extracellular matrix of chondrocytes in the processes of regeneration both in vitro and in vivo [19]. It is suspected that CHs improve bone formation and may be associated with the absorption of collagen-derived peptides thus affecting bone metabolism. After oral administration of collagen-derived peptides, some collagen derived di- or tripeptides can be detected in blood [20]. Furthermore, collagen-derived peptides stimulate osteoblast growth and differentiation, and reduce osteoclast differentiation to modulate bone formation [6].

In our previous study, there are collagen, peptides, polysaccharides, nucleosides, and nucleobases in EDC, however, the contents of polysaccharides, nucleosides, and nucleobases are very low. Compounds including 17 nucleosides and nucleobases were detected in EDC powder, and the total content was ranging from $6 \mu \mathrm{g} / \mathrm{g}$ to $49 \mu \mathrm{g} / \mathrm{g}$ [1]. The content of polysaccharides in EDCF is very low, which cannot be detected. In the present study, EDCF showed good efficacy and we speculate that other low molecular compound such as nucleosides, nucleobases or oligosaccharides might not be the active compound in EDCF, but the collagen derived peptides.

According to the traditional processing method, EDC is extracted with boiling water rather than enzymatic hydrolysis. We speculate that protein fragments or non-specific generated peptides could be obtained by non-specific proteolysis during extraction. As shown in Figure 5, hydrolyzed peptides did not show site-specific properties, and peptides were mainly generated from the seven "hot regions". However, based on the present study, it cannot be excluded that these peptides already exist naturally in the EDC sample before extraction, and we will try to clarify it in further studies. Based on the characterization analysis of the EDCF in the present study, the peptides in EDCF were probably generated from EDC collagen fragments as chemical cleavage during extraction. Furthermore, taking up these peptides could help to improve bone metabolism both in vitro and in vivo. In addition, most peptides in the EDCF were lower than $2 \mathrm{kDa}$, which means that it might be easier to obtain EDC peptides with MWs from 793.4 to 2000 Da by a non-specific hydrolysis process. Peptides with MWs lower than $2 \mathrm{kDa}$ may have more chance of crossing the biological membrane and exerting biological effects.

\section{Materials and Methods}

\subsection{Chemicals and Materials}

The samples of EDC were acquired from the Elaphuri Davidiani National Nature Reserve in Jiangsu Dafeng, and authenticated by Professor Jin-ao Duan from Nanjing University of Chinese Medicine. The EDC samples were then pulverized to obtain a fine powder.

Formic acid was obtained from Sigma-Aldrich Chemical Co., Ltd. (St. Louis, MO, USA). LC-grade methanol and acetonitrile were purchased from Merck KGaA (Darmstadt, Germany). Ultra-high purity water was prepared using a Millipore-Q system (Millipore Corporation, Billerica, MA, USA).

Vitamin C, dexamethasone and type I collagenase were purchased from Sigma-Aldrich Chemical Co., Ltd. MEM $\alpha$ basic $(1 \times)$, fetal bovine serum (FBS), Tris-EDTA buffer (TE), and $\alpha$-modified Eagle's medium- $\alpha$ ( $\alpha$-MEM) were purchased from Gibco Co., Ltd. (Grand Island, NY, USA). The PrimeScript ${ }^{\mathrm{TM}}$ RT Reagent Kit with a gDNA Eraser was purchased from TaKaRa Co., Ltd. (Otsu, Japan).

\subsection{Preparation of EDC Extract and Ultrafiltration}

EDC was cut into small pieces and subsequently pulverized to a fine powder. The powder $(200 \mathrm{~g})$ was decocted twice with $10 \times$ quantities of water $(2 \mathrm{~L})$ for $6 \mathrm{~h}$. The extract was filtered and the solvent was evaporated using a rotary evaporator at $60{ }^{\circ} \mathrm{C}$; then it was lyophilized and the resulting powdered EDC extract was stored at $-20^{\circ} \mathrm{C}$.

Ultrafiltration was performed using membranes with $3 \mathrm{kDa}$ cut-off values on a Mini Pellicon Ultrafiltration system (Millipore Corporation). The EDC extract prepared as above was redissolved in pure water, filtered through a $0.45 \mu \mathrm{m}$ nylon syringe filter and separated into two fractions. 
The fraction with a molecular weight lower than $3 \mathrm{kDa}$ was collected, lyophilized, and stored at $-20{ }^{\circ} \mathrm{C}$. The lyophilized EDCF was re-dissolved in $0.9 \%$ sterile saline as needed.

\subsection{Measurement of the Effects of EDC on Osteoblasts}

\subsubsection{Cell Culture}

The isolation and culture of osteoblasts was performed according to a previously described method [21] with some modifications. Postnatal day 1 rats were decapitated to collect calvarias. Briefly, tissues were sequentially digested with $1 \%$ trypsin for $10 \mathrm{~min}, 0.2 \%$ collagenase for $20 \mathrm{~min}$, and finally freshly prepared $0.2 \%$ collagenase for a further $40 \mathrm{~min}$. After digestion, the supernatant was collected and centrifuged for $5 \mathrm{~min}$ at $200 \times \mathrm{g}$. Osteoblasts were re-suspended and maintained in $\alpha$-MEM, supplemented with 10\% FBS, penicillin (100 U/mL), streptomycin $(100 \mu \mathrm{g} / \mathrm{mL})$, and $2 \mathrm{mM}$ L-glutamine in a humidified atmosphere of $5 \% \mathrm{CO}_{2}$ at $37^{\circ} \mathrm{C}$. The medium was changed every 3 days. Reagents for cell cultures were purchased from Invitrogen (Invitrogen Corporation, Carlsbad, CA, USA) The experiments on animals have been approved by the Animal Experimentation Ethics Committee of Nanjing University of Chinese Medicine and conformed to the guidelines of the "Principles of Laboratory Animal Care" (NIH publication No. 80-23, revised 1996). Effort was made to minimize the number and suffering of the animals.

\subsubsection{MTT Cell Viability Assay}

Cell viability was assessed using a modified MTT assay. Briefly, osteoblasts were seeded in six-well plates at a final concentration of $1 \times 10^{4}$ cells per well. After $24 \mathrm{~h}$, the medium was removed. Differentiation was induced by treatment with vitamin $C(100 \mu \mathrm{M})$ and dexamethasone $(20 \mathrm{nM})$. The cells were rinsed with phosphate-buffered saline (PBS) and incubated with EDCF at different concentrations $(10,30$, and $100 \mu \mathrm{g} / \mathrm{mL}$ ) and dexamethasone + vitamin C (Dex + Vit. C) for $24 \mathrm{~h}$. After removing the medium and washing with PBS twice, the cells were treated with MTT solution $(5 \mathrm{mg} / \mathrm{mL}$ in PBS) in medium without phenol red and serum. The cells were stored in a humidified atmosphere of $5 \% \mathrm{CO}_{2}$ at $37^{\circ} \mathrm{C}$. The liquid was then removed and dimethyl sulfoxide $(150 \mu \mathrm{L})$ was added. Absorbance at $570 \mathrm{~nm}$ was read in a microplate spectrophotometer (Tecan GENios, Tecan Trading AG, Männedorf, Switzerland).

\subsubsection{Real Time Quantitative PCR}

Differentiation was induced by treatment with vitamin $C(100 \mu \mathrm{M})$ and dexamethasone $(20 \mathrm{nM})$. After $24 \mathrm{~h}$ exposure to EDCF at different concentrations (10, 30, and $100 \mu \mathrm{g} / \mathrm{mL}$ ) and Dex + Vit. C, osteoblasts were harvested and rinsed twice using ice-cold PBS. Total RNA was extracted and transcribed into cDNA using Trizol and a reverse transcription kit (TaKaRa) according to the manufacturer's protocol. Real-time PCRs of COL1A1 and rat osteonectin were performed using equal amounts of reverse-transcribed products and carried out using SYBR Premix Ex Taq (TaKaRa). All data were normalized to the expression of the endogenous reference gene glyceraldehyde-3-phosphate dehydrogenase $(G A P D H)$. The following primers were used for qPCR amplifications: COL1A1 (5'-TCCGCCGATGTCGCTATC-3' and $5^{\prime}$-CAAGTTCCGGTGTGACTCGT- $\left.3^{\prime}\right)$; rat osteonectin $\left(5^{\prime}\right.$-GAA GAGATGGTGGCGGAG-3' ${ }^{\prime}$ and $5^{\prime}$-ACAGGCAGGGGGCAATGATTTG-3'); GAPDH (5'-GGTGAAGG TCGGTGTGAACG-3' ${ }^{\prime}$ and $5^{\prime}$-CTCGCTCCTGGAAGATGGTG-3'). The real-time PCR was performed in a $20 \mathrm{~mL}$ reaction system by an ABI Prism 7500 sequence amplification system (AB Sciex, Concord, ON, Canada). The relative levels of transcript expression were quantified using the $\Delta \Delta \mathrm{Ct}$ method.

\subsection{Peptide Characterization by Nano-LC-MS/MS}

All samples were analyzed by the Dionex 3000 nano-LC system tandem LTQ-Orbitrap Velos Pro (Thermo Fisher Scientific, Waltham, MA, USA) with HCD. Lyophilized EDCF was re-dissolved in $0.1 \%$ TFA, and was desalted by SepPak C18. Desalted EDCF sample was dried by centrifugal 
concentration. Then EDCF sample was re-dissolved in acetonitrile/formic acid/water (2/0.2/98, $v / v / v)$, and five microliters EDCF sample was loaded onto a self-packed $5 \mu \mathrm{m}$ Reprosil C18AQ column $(75 \mu \mathrm{m} \times 150 \mathrm{~mm})$. The mobile phase consisted of acetonitrile/formic acid/water $(2 / 0.2 / 98, v / v / v)$ for buffer A and acetonitrile/formic acid/water (80/0.2/20, $v / v / v)$ for buffer B. Processed samples were analyzed using a 150 min gradient from $2 \%$ to $30 \%$ of B. The LTQ-Orbitrap was operated in data-dependent acquisition mode to automatically alternate between a full scan $(\mathrm{m} / z$ 300-2000) in the Orbitrap and HCD MS/MS scans in the linear ion trap. Helium was used as the collision gas for HCD. The normalized collision energy was 35\% and the activation time was $30 \mathrm{~ms}$. Unless otherwise stated, three replicate measurements were made at each MS setting. Data acquisition was controlled by Xcalibur 2.0.7 and Tune 2.4 software (Thermo Fisher Scientific).

All MS/MS spectra were extracted with Xcalibur (version 2.0.7.) and analyzed using PEAKS 7.5 (Bioinformatics Solutions Inc., Waterloo, ON, Canada) which was set up to search the Pecora_uniprot-taxonomy_35500 database (downloaded on the 28 July 2015). Spectra were also searched against an equal number of decoy sequences to estimate the false discovery rate as described previously [22]. The specified enzyme was chosen as non-enzyme and up to two missed cleavages were allowed. Oxidation of methionine $(+15.9949)$ and acetylation of the protein N-terminus $(+42.0106)$ were specified as variable modifications. All other parameters were default settings, including a fragment ion tolerance of $0.5 \mathrm{Da}$ and a maximum precursor ion tolerance of $6 \mathrm{ppm}$ after recalibration. Identification of peptides by de novo sequence was filtered to curate a dataset with a value for the Average Local Confidence (ALC) score larger than 90\%.

\section{Conclusions}

In conclusion, the current study demonstrated that the fraction of EDC aqueous extract with a MW lower than $3 \mathrm{kDa}$ increased the viability of osteoblasts and increased the mRNA levels of osteonectin and COL1A1. Furthermore, a workflow for the analysis of peptides in EDCF was developed, and a total of 272 peptides were identified. The non-specific hydrolysis peptides of EDCF were described and a protocol was established to investigate the complex mixture from $\mathrm{CHs}$ and elucidate the process of peptides formation. The present investigation elucidated complex components derived from $\mathrm{CHs}$ using a time-saving and labor-saving nano-LC MS/MS analysis strategy.

Supplementary Materials: Supplementary materials can be accessed at: http:/ /www.mdpi.com/1420-3049/22/ 1/166/s1.

Acknowledgments: This work was supported by the National Natural Science Foundation of China (81274017 and 81202861), the Priority Academic Program Development of Jiangsu Higher Education Institutions, PAPD (ysxk-2010), the Open Project Program of National First-Class Key Discipline for Traditional Chinese Medicine of Nanjing University of Chinese Medicine (2011ZYX1-005), Jiangsu Qinlan Project and Jiangsu “333” Project.

Author Contributions: Rui Liu, Dawei Qian, Yue Zhu and Jin-ao Duan designed the experiments; Yanjuan Zhai, Zhenhua Zhu performed the experiments; Yunru Peng and Zhen Ouyang analyzed the data; Yuhua Ding contributed EDC materials; Yanjuan Zhai and Rui Liu wrote the paper.

Conflicts of Interest: The authors declare no conflict of interest.

\section{References}

1. Jiang, Z.; Harris, R.B. Elaphurus Davidianus, The IUCN Red List of Threatened Species. Version 2016-3. Available online: http:/ /www.iucnredlist.org (accessed on 21 December 2016).

2. Li, F.T.; Duan, J.A.; Qian, D.W.; Guo, S.; Ding, Y.H.; Liu, X.H.; Qian, Y.F.; Peng, Y.R.; Ren, Y.J.; Chen, Y. Comparative analysis of nucleosides and nucleobases from different sections of Elaphuri Davidiani Cornu and Cervi Cornu by UHPLC-MS/MS. J. Pharm. Biomed. Anal. 2013, 83, 10-18. [CrossRef] [PubMed]

3. Meng, H.Y.; Qu, X.B.; Li, N.; Yuan, S.; Lin, Z. Effects of pilose antler and antler glue on osteoporosis of ovariectomized rats. J. Chin. Med. Mater. 2009, 32, 179-182.

4. Qin, H.B.; Yang, C.Y.; Zhu, Q.; Zhu, Y.F. Effect of ethanol extract from Elaphuri Davidiani Cornu on anti-aging in mice. Chin. Tradit. Pat. Med. 2004, 26, 322-324. 
5. Yang, Z.Y.; Qin, H.B.; Cheng, H.L.; Zhu, Q. Effects of elk antlers ethanoic fluid extract on behavior and immune function of aging model mice. Chin. J. TCM Pharm. 2010, 25, 221-225.

6. Guillerminet, F.; Beaupied, H.; Fabien-Soulé, V.; Tomé, D.; Benhamou, C.L.; Roux, C.; Blais, A. Hydrolyzed collagen improves bone metabolism and biomechanical parameters in ovariectomized mice: An in vitro and in vivo study. Bone 2010, 46, 827-834. [CrossRef] [PubMed]

7. Nomura, Y.; Oohashi, K.; Watanabe, M.; Kasugai, S. Increase in bone mineral density through oral administration of shark gelatin to ovariectomized rats. Nutrition 2005, 21, 1120-1126. [CrossRef] [PubMed]

8. Rolf, H.J.; Enderle, A. Hard fallow deer antler: A living bone till antler casting? Anat. Rec. 1999, 255, 69-77. [CrossRef]

9. Léonard, A.; Guiot, L.P.; Pirard, J.P.; Crine, M.; Balligand, M.; Blacher, S. Non-destructive characterization of deer (Cervus Elaphus) antlers by X-ray microtomography coupled with image analysis. J. Microsc. 2007, 225, 258-263. [CrossRef] [PubMed]

10. Buckley, M.; Collins, M.; Thomas-Oates, J.; Wilson, J.C. Species identification by analysis of bone collagen using matrix-assisted laser desorption/ionisation time-of-flight mass spectrometry. Rapid Commun. Mass Spectrom. 2009, 23, 3843-3854. [CrossRef] [PubMed]

11. Kim, H.K.; Kim, M.G.; Leem, K.H. Osteogenic activity of collagen peptide via ERK/MAPK pathway mediated boosting of collagen synthesis and its therapeutic efficacy in osteoporotic bone by back-scattered electron imaging and microarchitecture analysis. Molecules 2013, 18, 15474-15489. [CrossRef] [PubMed]

12. Wolters, D.A.; Washburn, M.P.; Yates, J.R., 3rd. An automated multidimensional protein identification technology for shotgun proteomics. Anal. Chem. 2001, 73, 5683-5690. [CrossRef] [PubMed]

13. Hu, C.H.; Yao, C.H.; Chan, T.M.; Huang, T.L.; Sen, Y.; Huang, C.Y.; Ho, C.Y. Effects of different concentrations of collagenous peptide from fish scales on osteoblast proliferation and osteoclast pesorption. Chin. J. Physiol. 2016, 59, 191-201. [CrossRef] [PubMed]

14. Bella, J. Collagen structure: New tricks from a very old dog. Biochem. J. 2016, 473, 1001-1025. [CrossRef] [PubMed]

15. Liu, R.; Wang, M.; Duan, J.A.; Guo, J.M.; Tang, Y.P. Purification and identification of three novel antioxidant peptides from Cornu Bubali (water buffalo horn). Peptides 2010, 31, 786-793. [CrossRef] [PubMed]

16. Piovesana, S.; Capriotti, A.L.; Cavaliere, C.; La Barbera, G.; Samperi, R.; Zenezini Chiozzi, R.; Laganà, A. Peptidome characterization and bioactivity analysis of donkey milk. J. Proteom. 2015, 119, 21-29. [CrossRef] [PubMed]

17. Wu, J.; Fujioka, M.; Sugimoto, K.; Mu, G.; Ishimi, Y. Assessment of effectiveness of oral administration of collagen peptide on bone metabolism in growing and mature rats. J. Bone Miner. Metab. 2004, 22, 547-553. [CrossRef] [PubMed]

18. De Almeida Jackix, E.; Cúneo, F.; Amaya-Farfan, J.; de Assunção, J.V.; Quintaes, K.D. A food supplement of hydrolyzed collagen improves compositional and biodynamic characteristics of vertebrae in ovariectomized rats. J. Med. Food 2010, 13, 1385-1390. [CrossRef] [PubMed]

19. Oesser, S.; Seifert, J. Stimulation of type II collagen biosynthesis and secretion in bovine chondrocytes cultured with degraded collagen. Cell Tissue Res. 2003, 311, 393-399. [PubMed]

20. Iwai, K.; Hasegawa, T.; Taguchi, Y.; Morimatsu, F.; Sato, K.; Nakamura, Y.; Higashi, A.; Kido, Y.; Nakabo, Y.; Ohtsuki, K. Identification of food-derived collagen peptides in human blood after oral ingestion of gelatin hydrolysates. J. Agric. Food Chem. 2005, 53, 6531-6536. [CrossRef] [PubMed]

21. Guo, A.J.; Choi, R.C.; Cheung, A.W.; Chen, V.P.; Xu, S.L.; Dong, T.T.; Chen, J.J.; Tsim, K.W. Baicalin, a flavone, induces the differentiation of cultured osteoblasts. J. Biol. Chem. 2011, 286, 27882-27893. [CrossRef] [PubMed]

22. Dietrich, M.A.; Arnold, G.J.; Nynca, J.; Fröhlich, T.; Otte, K.; Ciereszko, A. Characterization of carp seminal plasma proteome in relation to blood plasma. J. Proteom. 2014, 98, 218-232. [CrossRef] [PubMed]

Sample Availability: Samples of all compounds are available from the authors.

(C) 2017 by the authors; licensee MDPI, Basel, Switzerland. This article is an open access article distributed under the terms and conditions of the Creative Commons Attribution (CC BY) license (http://creativecommons.org/licenses/by/4.0/). 